\title{
Six-DOF Impedance Control of Dual-Arm Cooperative Manipulators
}

\author{
Fabrizio Caccavale, Senior Member, IEEE, Pasquale Chiacchio, Alessandro Marino, \\ and Luigi Villani, Senior Member, IEEE
}

\begin{abstract}
In this paper, the problem of impedance control of dual-arm cooperative manipulators is studied. A general impedance control scheme is adopted, which encompasses a centralized impedance control strategy, aimed at conferring a compliant behavior at the object level, and a decentralized impedance control, enforced at the end-effector level, aimed at avoiding large internal loading of the object. Remarkably, the mechanical impedance behavior is defined in terms of geometrically consistent stiffness. The overall control scheme is based on a twoloop arrangement, where a simple proportional integral derivative inner motion loop is adopted for each manipulator, while an outer loop, using force and moment measurements at the robots wrists, is aimed at imposing the desired impedance behaviors. The developed control scheme is experimentally tested on a dual-arm setup composed of two 6-DOF industrial manipulators carrying a common object. The experimental investigation concerns the four different controller configurations that can be achieved by activating/deactivating the single impedance controllers.
\end{abstract}

Index Terms-Control, cooperative manipulators, robotics.

\section{INTRODUCTION}

$\mathbf{T}$ HE ADOPTION of cooperative manipulators is often required to execute a wide class of tasks, such as carrying large or heavy payloads and mating of mechanical parts [1]. When a cooperative multiarm system is employed for the manipulation of a common object, it is important to control both the absolute motion of the object and the internal stresses. To this aim, the mappings between forces and velocities at the end effector of each manipulator and their counterparts at the manipulated object are to be considered [2]. In this framework, several strategies have been proposed to control both absolute motion and internal forces (see, e.g., [3]-[6]) a recent control framework for cooperative systems is based on the so-called synchronization control [7], [8], where the control problem is formulated in terms of suitably defined motion synchronization errors between the manipulators involved in the cooperative

Manuscript received February 19, 2008; revised May 9, 2008. Current version published October 8, 2008. Recommended by Technical Editor S. Chiaverini. This work was supported in part by the DEXMART large-scale integrating project, funded by the European Community's Seventh Framework Programme under Grant Agreement ICT-216239 and in part by MIUR (Italian Ministry of Research) under Grant PRIN 2007.

F. Caccavale and A. Marino are with the Dipartimento di Ingegneria e Fisica dell'Ambiente, Università degli Studi della Basilicata, 85100 Potenza, Italy (e-mail: fabrizio.caccavale@unibas.it; alessandro.marino@unibas.it).

P. Chiacchio is with the Dipartimento di Ingegneria dell'Informazione e di Ingegneria Elettrica, Università degli Studi di Salerno, 84084 Fisciano, Italy (e-mail: pchiacchio@unisa.it).

L. Villani is with the Dipartimento di Informatica e Sistemistica, Università degli Studi di Napoli Federico II, 80125 Naples, Italy (e-mail: luigi.villani@ unina.it).

Color versions of one or more of the figures in this paper are available online at http://ieeexplore.ieee.org.

Digital Object Identifier 10.1109/TMECH.2008.2002816 task. Interesting contributions [9]-[11] have recast the problem of cooperative robots control in the framework of intelligent control. Also, the control of cooperative manipulators via partial state feedback has been considered in [12]. Finally, the extension of cooperative manipulation concepts to cooperative transportation via mobile robotic platforms is worth mentioning (see, e.g., [13]).

Most of the earlier approaches can be classified as force/motion control schemes. An alternative method can be pursued based on the well-known impedance concept [14]. Impedance control schemes for cooperative manipulators have been proposed for the control of either object/environment interaction forces [17] or internal forces [18].

Namely, when the held object interacts with the environment, large contact forces may arise if the planned trajectory is not consistent with the geometry of the environment. In order to achieve bounded contact forces, an impedance behavior can be enforced between the object's position/orientation displacements and the contact force/moment (external impedance). This approach is of a centralized type, since the object motion variables depend on the configuration of all the manipulators.

On the other hand, even when the object/environment interaction does not take place, the interaction between the manipulators and the object may lead to internal forces and moments, i.e., mechanical stresses that do not contribute to the object's motion, and may cause damage to the system and overloading of the actuators. To counteract building of large values of internal forces, an impedance behavior can be enforced between the position/orientation displacements of each manipulator and the end-effector force/moment, contributing solely to the internal loading of the object (internal impedance). This approach is of decentralized type, since it involves the motion variables of each manipulator separately.

The two impedance approaches have been applied separately [17], [18]. In this paper, by following the guidelines in [19], they have been combined in a unique control framework, aimed at controlling both the contact forces due to the object/environment interaction (external impedance) and the internal forces due to the manipulators/object interaction (internal impedance). The scheme allows individual activation/deactivation of external and internal impedance; hence, the user might choose to keep one of (or both) the impedance controllers or decide to deactivate both; in the latter case, a purely positional control strategy is adopted.

Moreover, in this paper, the impedance control scheme is reformulated according to the concept of geometrically consistent stiffness and is experimentally tested. Namely, the equations defining the impedance behavior, at both the object (external 
impedance) and end-effectors level (internal impedance), are derived by considering a rigorous and geometrically meaningful definition of a 6-DOF mechanical stiffness, i.e., encompassing both translational and rotational displacements [20].

The overall control strategy is based on a two-loop arrangement, where a simple proportional-integral-derivative (PID) inner motion loop at the joints is adopted for each manipulator, while an outer loop, using force and moment measurements at the robots wrists, is aimed at imposing the assigned impedance behaviors. Remarkably, the use of standard PID controllers makes the approach more attractive from an industrial point of view, since industrial robotic units usually adopt simple linear motion controllers of PID type.

The theoretical derivation of the proposed impedance scheme is based on the rigid object assumption. However, the experiments show that the scheme performs well, even in the presence of deformable manipulated objects.

Finally, a major contribution is given by the experimental testing of the proposed impedance scheme on an industrial robotic setup. In fact, just a few contributions in the literature (e.g., [21]) tackle the problem of the application of cooperative manipulation in an industrial context. Namely, a goal of this paper is that of demonstrating the performance of impedance control even in the presence of mechanical limitations typical of industrial setups, due to, e.g., friction, backlash, slipping at grasp points, and other phenomena difficult to model. The setup is composed of two 6-DOF COMAU Smart-3 S robots grasping a large cardbox; interaction of the box with a nonrigid surface (whose position and orientation are unknown to the controller) has been imposed in the experiments.

\section{ModelinG}

Consider a system of two six-joint manipulators, tightly grasping a common rigid object.

For each manipulator $(k=1,2)$, let $\Sigma_{k}$ denote a frame attached to the end effector, whose origin and orientation are characterized by the $(3 \times 1)$ position vector $\boldsymbol{p}_{k}$ and the $(3 \times 3)$ rotation matrix $\boldsymbol{R}_{k}$, respectively. Let $\mathcal{Q}_{k}=\left\{\eta_{k}, \boldsymbol{\epsilon}_{k}\right\}$ be the unit quaternion corresponding to $\boldsymbol{R}_{k}$ [22], [23]. Since the grasp is tight and the object is rigid, the relative orientation between $\Sigma_{1}$ and $\Sigma_{2}$ is constant and can be set so that $\boldsymbol{R}_{1}=\boldsymbol{R}_{2}$. Let also $\boldsymbol{v}_{k}=\left[\begin{array}{ll}\dot{\boldsymbol{p}}_{k}^{T} & \boldsymbol{\omega}_{k}^{T}\end{array}\right]^{T}$ be the $(6 \times 1)$ end-effector (linear and angular) velocity vector.

Further, consider a frame $\Sigma_{e}$ attached to the object; the origin $\boldsymbol{p}_{e}$ is chosen so as to coincide with the object's center of mass, while the orientation is chosen so as to coincide with those of the two end-effector frames, i.e., $\boldsymbol{R}_{e}=\boldsymbol{R}_{1}=\boldsymbol{R}_{2}$. Let also $\mathcal{Q}_{e}$ be the unit quaternion corresponding to $\boldsymbol{R}_{e}$.

All quantities are expressed in the common base frame $\Sigma$. Hereafter, a superscript will denote the frame to which a quantity (vector or matrix) is referred; the superscript will be dropped whenever a quantity is referred to the base frame.

The dynamics of the two manipulators can be written in compact form as

$$
\boldsymbol{M}(\boldsymbol{q}) \ddot{\boldsymbol{q}}+\boldsymbol{C}(\boldsymbol{q}, \dot{\boldsymbol{q}}) \dot{\boldsymbol{q}}+\boldsymbol{d}(\boldsymbol{q}, \dot{\boldsymbol{q}})+\boldsymbol{g}(\boldsymbol{q})=\boldsymbol{\tau}-\boldsymbol{J}^{T}(\boldsymbol{q}) \boldsymbol{h}
$$

where the matrices are block diagonal, e.g., $\quad \boldsymbol{M}=$ blockdiag $\left(\boldsymbol{M}_{1}, \boldsymbol{M}_{2}\right), \boldsymbol{J}=\operatorname{blockdiag}\left(\boldsymbol{J}_{1}, \boldsymbol{J}_{2}\right)$, and the vectors are stacked, e.g., $\boldsymbol{g}=\left[\begin{array}{ll}\boldsymbol{g}_{1}^{T} & \boldsymbol{g}_{2}^{T}\end{array}\right]^{T}$. For each manipulator $(k=1,2), \boldsymbol{M}_{k}$ is the $(6 \times 6)$ symmetric positive-definite inertia matrix, $\boldsymbol{C}_{k} \dot{\boldsymbol{q}}_{k}$ is the $(6 \times 1)$ vector of Coriolis and centrifugal forces, $\boldsymbol{g}_{k}$ is the $(6 \times 1)$ vector of gravitational forces, $\boldsymbol{J}_{k}$ is the $(6 \times 6)$ Jacobian matrix, the vector $\boldsymbol{\tau}_{k}$ represents the joint torques, and $\boldsymbol{h}_{k}=\left[\begin{array}{ll}\boldsymbol{f}_{k}^{T} & \boldsymbol{\mu}_{k}^{T}\end{array}\right]^{T}$ is the $(6 \times 1)$ vector of forces and moments (wrench) acting at the end effector of the $k$ th manipulator. The term $\boldsymbol{d}$ represents a vector of disturbance terms, due to inaccurate modeling (e.g., joint friction torques) and/or external disturbances.

The dynamics of the object can be described by the equations

$$
\boldsymbol{M}_{e} \dot{\boldsymbol{v}}_{e}^{e}+\boldsymbol{C}\left(\boldsymbol{v}_{e}^{e}\right) \boldsymbol{v}_{e}^{e}+\boldsymbol{g}_{e}^{e}=\boldsymbol{h}_{e}^{e}-\boldsymbol{h}_{\mathrm{env}}^{e}
$$

where

$$
\begin{aligned}
\boldsymbol{M}_{e} & =\left[\begin{array}{cc}
m_{e} \boldsymbol{I} & \boldsymbol{O} \\
\boldsymbol{O} & \boldsymbol{J}_{e}^{e}
\end{array}\right] \\
\boldsymbol{C}_{e}\left(\boldsymbol{v}_{e}^{e}\right) & =\left[\begin{array}{cc}
m_{e} \boldsymbol{S}\left(\boldsymbol{\omega}_{e}^{e}\right) & \boldsymbol{O} \\
\boldsymbol{O} & \boldsymbol{S}\left(\boldsymbol{\omega}_{e}^{e}\right) \boldsymbol{J}_{e}^{e}
\end{array}\right] \\
\boldsymbol{g}_{e}^{e} & =\left[\begin{array}{c}
-m_{e} \boldsymbol{g}_{0}^{e} \\
\boldsymbol{0}
\end{array}\right]
\end{aligned}
$$

$\boldsymbol{S}(\cdot)$ is the skew-symmetric matrix operator performing the cross product, $\boldsymbol{v}_{e}^{e}=\left[\dot{\boldsymbol{p}}_{e}^{e T} \boldsymbol{\omega}_{e}^{e T}\right]^{T}$ is the vector expressing the linear and angular velocities of $\Sigma_{e}, m_{e}$ is the object mass, $\boldsymbol{J}_{e}^{e}$ is the object's inertia tensor referred to $\Sigma_{e}, \boldsymbol{g}_{0}^{e}$ is the vector of gravitational forces, $\boldsymbol{h}_{e}^{e}=\left[\begin{array}{ll}\boldsymbol{f}_{e}^{e T} & \boldsymbol{\mu}_{e}^{e T}\end{array}\right]^{T}$ is the wrench exerted by the manipulators on the object (i.e., the so-called external forces), and $\boldsymbol{h}_{\mathrm{env}}^{e}=\left[\begin{array}{ll}\boldsymbol{f}_{\mathrm{env}}^{e T} & \boldsymbol{\mu}_{\mathrm{env}}^{e T}\end{array}\right]^{T}$ is the wrench exerted by the object on the environment; all the earlier quantities are expressed in the frame $\Sigma_{e}$.

Since the grasp is tight, each end effector can exert both a force $\boldsymbol{f}_{k}$ and a moment $\boldsymbol{\mu}_{k}$ on the object at the contact point. The mapping of the contact force vector

$$
\boldsymbol{h}=\left[\begin{array}{l}
\boldsymbol{h}_{1} \\
\boldsymbol{h}_{2}
\end{array}\right]
$$

into the $(6 \times 1)$ force vector $\boldsymbol{h}_{E}$ is given by

$$
\boldsymbol{h}_{e}=\left[\begin{array}{cccc}
\boldsymbol{I}_{3} & \boldsymbol{O}_{3} & \boldsymbol{I}_{3} & \boldsymbol{O}_{3} \\
\boldsymbol{S}\left(\boldsymbol{r}_{1}\right) & \boldsymbol{I}_{3} & \boldsymbol{S}\left(\boldsymbol{r}_{2}\right) & \boldsymbol{I}_{3}
\end{array}\right]\left[\begin{array}{l}
\boldsymbol{h}_{1} \\
\boldsymbol{h}_{2}
\end{array}\right]=\boldsymbol{W h}
$$

where $\boldsymbol{W}$ is the grasp matrix, $\boldsymbol{O}_{l}$ denotes the $(l \times l)$ null matrix, $\boldsymbol{I}_{l}$ denotes the $(l \times l)$ identity matrix, and $\boldsymbol{r}_{k}$ is the $(3 \times 1)$ vector from the $k$ th end effector to the point fixed on the object (i.e., $\boldsymbol{r}_{k}$ is the so-called virtual stick [2]).

The matrix $\boldsymbol{W}$ is full-row rank, then, for a given $\boldsymbol{h}_{e}$, the inverse solution to (3) is given by

$$
\boldsymbol{h}=\boldsymbol{W}^{\dagger} \boldsymbol{h}_{e}+\boldsymbol{V} \boldsymbol{h}_{i}=\boldsymbol{h}_{E}+\boldsymbol{h}_{I}
$$

where $\boldsymbol{W}^{\dagger}$ denotes a pseudoinverse of $\boldsymbol{W}, \boldsymbol{V}$ is a full-column rank matrix spanning the null space of $\boldsymbol{W}$, and $\boldsymbol{h}_{i}$ represents the vector of internal forces, i.e., the forces that do not contribute to the motion of the object, but represent mechanical stresses applied to the object. They may be caused by the forces applied to the object attempting to violate the grasp constraints, 
e.g., the forces due to the object/environment interaction and/or squeezing and bending forces applied by the manipulators (due to end-effector trajectories not consistent with the closed-chain constraints). Internal forces may be usefully exploited only in special cases (e.g., in order to ensure a firm grasp) whereas, in general, they must be avoided.

It has been recognized in [24] that the use in (4) of a generic pseudoinverse of $\boldsymbol{W}$, e.g., the Moore-Penrose pseudoinverse, may lead to internal stresses even if $\boldsymbol{h}_{i}=\mathbf{0}$; to avoid this, $\boldsymbol{W}^{\dagger}$ must be properly chosen.

The vector

$$
\boldsymbol{h}_{E}=\left[\begin{array}{l}
\boldsymbol{h}_{E 1} \\
\boldsymbol{h}_{E 2}
\end{array}\right]=\boldsymbol{W}^{\dagger} \boldsymbol{W} \boldsymbol{h}
$$

represents the end-effector forces balancing the object's dynamics and the contact forces due to the interaction with the environment, while

$$
\boldsymbol{h}_{I}=\left[\begin{array}{l}
\boldsymbol{h}_{I 1} \\
\boldsymbol{h}_{I 2}
\end{array}\right]=\boldsymbol{V} \boldsymbol{h}_{i}=\boldsymbol{V} \boldsymbol{V}^{\dagger} \boldsymbol{h}
$$

represents the vector of end-effector forces contributing to the sole internal forces.

According to the earlier description of the geometry of the grasp, the end-effector quantities corresponding to a given object's motion can be expressed as

$$
\left\{\begin{array}{l}
\boldsymbol{p}_{k}=\boldsymbol{p}_{e}+\boldsymbol{R}_{e} \boldsymbol{r}_{k}^{e} \\
\boldsymbol{R}_{k}=\boldsymbol{R}_{e} \\
\dot{\boldsymbol{p}}_{k}=\dot{\boldsymbol{p}}_{e}-\boldsymbol{S}\left(\boldsymbol{R}_{e} \boldsymbol{r}_{k}^{e}\right) \boldsymbol{\omega}_{e} \\
\boldsymbol{\omega}_{k}=\boldsymbol{\omega}_{e} \\
\ddot{\boldsymbol{p}}_{k}=\ddot{\boldsymbol{p}}_{e}-\boldsymbol{S}\left(\boldsymbol{\omega}_{e}\right) \boldsymbol{S}\left(\boldsymbol{R}_{e} \boldsymbol{r}_{k}^{e}\right) \boldsymbol{\omega}_{e}-\boldsymbol{S}\left(\boldsymbol{R}_{e} \boldsymbol{r}_{k}^{e}\right) \dot{\boldsymbol{\omega}}_{e} \\
\dot{\boldsymbol{\omega}}_{k}=\dot{\boldsymbol{\omega}}_{e}
\end{array}\right.
$$

with $k=1,2$. The previous equations define a set of mechanical constraints on the position and orientation of the manipulators end effectors, and thus they are always fulfilled during system's motion.

\section{INNER MOTION CONTROL LOOP}

In the remainder, an inner motion control loop is designed for each manipulator, which guarantees tracking of a reference end-effector frame $\Sigma_{k_{r}}$ having position $\boldsymbol{p}_{k_{r}}$ and orientation $\mathcal{Q}_{k_{r}}$ (extracted from the corresponding rotation matrix $\boldsymbol{R}_{k_{r}}$ ), as well as of a reference end-effector velocity $\boldsymbol{v}_{k_{r}}=\left[\begin{array}{ll}\dot{\boldsymbol{p}}_{k_{r}}^{T} & \boldsymbol{\omega}_{k_{r}}^{T}\end{array}\right]^{T}$.

With this purpose, a second-order closed loop inverse kinematics (CLIK) algorithm [25] is employed for each manipulator to compute the corresponding reference joints positions $\boldsymbol{q}_{k_{r}}$ and velocities $\dot{\boldsymbol{q}}_{k_{r}}$. Namely, given the reference position $\boldsymbol{p}_{k_{r}}$ and orientation $\mathcal{Q}_{k_{r}}$ trajectories, together with the corresponding velocities $\dot{\boldsymbol{p}}_{k_{r}}$ and $\boldsymbol{\omega}_{k_{r}}$ and accelerations $\ddot{\boldsymbol{p}}_{k_{r}}$ and $\dot{\boldsymbol{\omega}}_{k_{r}}$, the corresponding joints accelerations can be computed as

$$
\begin{gathered}
\ddot{\boldsymbol{q}}_{k_{r}}=\boldsymbol{J}_{k}^{-1}\left(\boldsymbol{q}_{k_{r}}\right)\left(\left[\begin{array}{l}
\ddot{\boldsymbol{p}}_{k_{r}}+k_{V p}\left(\dot{\boldsymbol{p}}_{k_{r}}-\dot{\boldsymbol{p}}_{k_{c}}\right)+k_{P p}\left(\boldsymbol{p}_{k_{r}}-\boldsymbol{p}_{k_{c}}\right) \\
\dot{\boldsymbol{\omega}}_{k_{r}}+k_{V o}\left(\boldsymbol{\omega}_{k_{r}}-\boldsymbol{\omega}_{k_{c}}\right)+k_{P o} \boldsymbol{\epsilon}_{r c}
\end{array}\right]\right. \\
\left.-\dot{\boldsymbol{J}}_{k}\left(\boldsymbol{q}_{k_{r}}, \dot{\boldsymbol{q}}_{k_{r}}\right) \dot{\boldsymbol{q}}_{k_{r}}\right)
\end{gathered}
$$

where $k_{V p}, k_{P p}, k_{V o}$, and $k_{P o}$ are positive gains.
The quantities $\boldsymbol{p}_{k_{c}}$ and $\mathcal{Q}_{k_{c}}$ are, respectively, the vector and the unit quaternion expressing the position and the orientation of the end-effector frame $\Sigma_{k_{c}}$ obtained from $\boldsymbol{q}_{k_{r}}$ by computing the direct kinematics of the manipulator; the same applies for the corresponding velocities $\dot{\boldsymbol{p}}_{k_{c}}$ and $\boldsymbol{\omega}_{k_{c}}$. The orientation error $\boldsymbol{\epsilon}_{k_{r} k_{c}}=\boldsymbol{R}_{k_{c}}^{k_{c}} \boldsymbol{\epsilon}_{k_{r} k_{c}}$ is obtained by expressing the mutual orientation of $\Sigma_{k_{r}}$ with respect to $\Sigma_{k_{c}}$ via the unit quaternion

$$
\begin{aligned}
\mathcal{Q}_{k_{r} k_{c}} & =\mathcal{Q}_{k_{r} k_{c}}^{-1} * \mathcal{Q}_{k_{r}}=\left\{\eta_{k_{r} k_{c}},{ }^{c} \boldsymbol{\epsilon}_{k_{r} k_{c}}\right\} \\
& =\left\{\cos \frac{\vartheta_{k_{r} k_{c}}}{2}, \sin \frac{\vartheta_{k_{r} k_{c}}}{2}{ }^{c} \boldsymbol{r}_{r c}\right\}
\end{aligned}
$$

where $(*)$ denotes the quaternion product and $(\cdot)^{-1}$ denotes the conjugate of the unit quaternion.

Once the joint reference trajectories are computed, the driving torques are generated via a standard PID control law

$$
\begin{aligned}
\boldsymbol{\tau}_{k}=\boldsymbol{G}_{P, k}\left(\boldsymbol{q}_{k_{r}}-\boldsymbol{q}_{k}\right) & +\boldsymbol{G}_{V, k}\left(\dot{\boldsymbol{q}}_{k_{r}}-\dot{\boldsymbol{q}}_{k}\right) \\
& +\boldsymbol{G}_{I, k} \int_{t_{0}}^{t}\left(\boldsymbol{q}_{k_{r}}(\sigma)-\boldsymbol{q}_{k}(\sigma)\right) d \sigma
\end{aligned}
$$

where $\boldsymbol{q}_{k}$ is the vector of the measured joints variables, and $\boldsymbol{G}_{P, k}, \boldsymbol{G}_{V, k}$, and $\boldsymbol{G}_{I, k}$ are diagonal and positive-definite matrix gains, to be tuned so as to provide accurate and fast tracking of reference trajectories as well as robustness to disturbances and uncertainties (see, e.g., [26] for a rigorous analysis of convergence of PID control for robot manipulators). Therefore, if control law (10) achieves accurate tracking of the reference trajectories, i.e., $\boldsymbol{q}_{k} \simeq \boldsymbol{q}_{k_{r}}$, it can be assumed that

$$
\begin{array}{lll}
\boldsymbol{p}_{k} \simeq \boldsymbol{p}_{k_{r}}, & \boldsymbol{R}_{k} \simeq \boldsymbol{R}_{k_{r}} \\
\dot{\boldsymbol{p}}_{k} \simeq \dot{\boldsymbol{p}}_{k_{r}}, & \boldsymbol{\omega}_{k} \simeq \boldsymbol{\omega}_{k_{r}} \\
\ddot{\boldsymbol{p}}_{k} \simeq \ddot{\boldsymbol{p}}_{k_{r}}, & & \dot{\boldsymbol{\omega}}_{k} \simeq \dot{\boldsymbol{\omega}}_{k_{r}} .
\end{array}
$$

Remarkably, the use of the standard PID controllers makes more attractive the approach from an industrial point of view, since industrial robotic units usually adopt simple linear motion controllers of PID type. Of course, the overall scheme will result in more performance if more sophisticated joints motion controllers are adopted (e.g., inverse dynamics control, adaptive or robust control strategies). Also, the adoption of inner position loops is standard in sensor-based control approaches (e.g., force/position control, visual servoing) for industrial robots.

The inner motion control loop represents the basis for the development of the two approaches analyzed in the following. Namely, the control problem for cooperative manipulation is formulated as that of computing suitable reference position and orientation trajectories for the inner control loop.

\section{IMPEDANCE CONTROL}

In this section, the impedance control scheme first proposed in [19] and depicted in Fig. 1 is reviewed and extended according to the concept of geometrically consistent stiffness. Let the desired object position $\boldsymbol{p}_{e_{d}}(t)$ and orientation $\mathcal{Q}_{e_{d}}(t)$ [extracted from $\boldsymbol{R}_{e_{d}}(t)$ ] be assigned with the associated linear and angular velocities and accelerations. Hereafter, the 


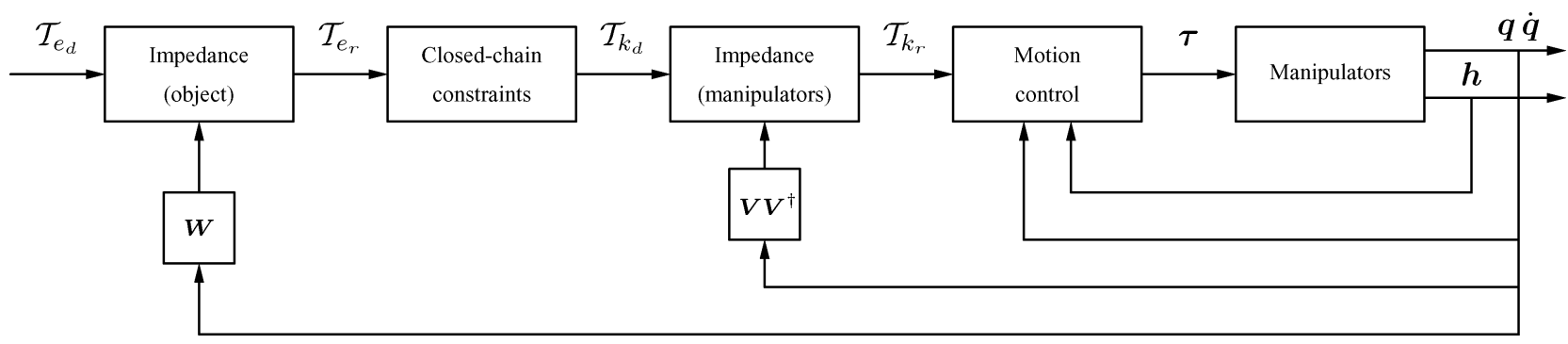

Fig. 1. Block diagram of the impedance control scheme.

set of variables defining the desired trajectory will be denoted by $\mathcal{T}_{e_{d}}=\left\{\boldsymbol{p}_{e_{d}}, \boldsymbol{R}_{e_{d}}, \dot{\boldsymbol{p}}_{e_{d}}, \boldsymbol{\omega}_{e_{d}}, \ddot{\boldsymbol{p}}_{e_{d}}, \dot{\boldsymbol{\omega}}_{e_{d}}\right\}$. If a purely positional control strategy is pursued, the reference trajectory for each manipulator $\mathcal{T}_{k_{r}}=\left\{\boldsymbol{p}_{k_{r}}, \boldsymbol{R}_{k_{r}}, \dot{\boldsymbol{p}}_{k_{r}}, \boldsymbol{\omega}_{k_{r}}, \ddot{\boldsymbol{p}}_{k_{r}}, \dot{\boldsymbol{\omega}}_{k_{r}}\right\}$ (with $\left.k_{r}=1,2\right)$ is generated from the desired trajectory $\mathcal{T}_{e_{d}}$ according to (7), and is input to the inner motion control loop.

If $\mathcal{T}_{k_{r}}$ is consistent with the geometry of the grasp, in the absence of interaction of the object with the environment, tracking of $\boldsymbol{p}_{e_{d}}, \mathcal{Q}_{e_{d}}$, and the corresponding velocity and acceleration is guaranteed by the inner motion control loop.

However, the adoption of a purely positional control strategy may lead to the build up of large forces (both external and internal).

Hence, in the following, an impedance control strategy is devised aimed at limiting both internal and contact forces.

When a robot manipulator interacts with other manipulators and/or with the environment, a suitable compliant behavior has to be ensured so as to achieve bounded interaction forces. This is typically achieved by enforcing an equivalent mass-damperspring behavior under the action of an external force and moment, that can be described by a mechanical impedance [14].

In order to derive the impedance equation, let us consider the mutual position and orientation between a desired frame $\Sigma_{d}$ and a reference frame $\Sigma_{r}$.

Namely, the mutual position vector is

$$
\Delta \boldsymbol{p}_{d r}=\boldsymbol{p}_{d}-\boldsymbol{p}_{r}
$$

while the mutual orientation of $\Sigma_{d}$ with respect to $\Sigma_{r}$ can be expressed in terms of the unit quaternion (corresponding to the mutual rotation matrix $\boldsymbol{R}_{d r}^{r}=\boldsymbol{R}_{r}^{T} \boldsymbol{R}_{d}$ )

$$
\begin{aligned}
\mathcal{Q}_{d r}=\mathcal{Q}_{r}^{-1} * \mathcal{Q}_{d} & =\left\{\eta_{d r}, \boldsymbol{\epsilon}_{d r}^{r}\right\} \\
& =\left\{\cos \frac{\vartheta_{d r}}{2}, \sin \frac{\vartheta_{d r}}{2} \boldsymbol{r}_{d r}^{r}\right\}
\end{aligned}
$$

where, as usual, $\mathcal{Q}_{r}$ and $\mathcal{Q}_{d}$ are the unit quaternions extracted, respectively, from $\boldsymbol{R}_{r}$ and $\boldsymbol{R}_{d}$.

\section{A. Mechanical Springs}

The basic concept of mechanical impedance is strongly connected to the concept of mechanical stiffness, which is, in turn, related to the extension of the concept of mechanical spring from the unidimensional to the spatial (i.e., 6-DOF) case.

Assuming that, at equilibrium, frames $\Sigma_{d}$ and $\Sigma_{r}$ coincide, the compliant behavior near the equilibrium can be described by the linear mapping

$$
\boldsymbol{h}_{r}^{r}=\boldsymbol{K} \boldsymbol{\delta} \boldsymbol{x}_{d r}^{r}=\left[\begin{array}{ll}
\boldsymbol{K}_{p} & \boldsymbol{K}_{c} \\
\boldsymbol{K}_{c}^{T} & \boldsymbol{K}_{o}
\end{array}\right] \boldsymbol{\delta} \boldsymbol{x}_{d r}^{r}
$$

where $\boldsymbol{h}_{r}^{r}$ is the elastic wrench applied to frame $\Sigma_{r}$, expressed in $\Sigma_{r}$, in the presence of an infinitesimal twist displacement $\boldsymbol{\delta}_{\boldsymbol{x}_{d r}^{r}}^{r}$ of frame $\Sigma_{d}$ with respect to frame $\Sigma_{r}$, expressed in frame $\Sigma_{r}$. The elastic wrench and the infinitesimal twist displacement in (13) can be expressed equivalently also in frame $\Sigma_{d}$, since $\Sigma_{d}$ and $\Sigma_{r}$ coincide at equilibrium. Therefore, $\boldsymbol{h}_{r}^{r}=\boldsymbol{h}_{r}^{d}$ and $\boldsymbol{\delta} \boldsymbol{x}_{d r}^{r}=\boldsymbol{\delta} \boldsymbol{x}_{d r}^{d}$; moreover, for the elastic wrench applied to the desired frame $\Sigma_{d}, \boldsymbol{h}_{r}^{r}=\boldsymbol{K} \boldsymbol{\delta} \boldsymbol{x}_{r d}^{d}=-\boldsymbol{h}_{d}^{d}$ as $\boldsymbol{\delta} \boldsymbol{x}_{r d}^{d}=-\boldsymbol{\delta} \boldsymbol{x}_{d r}^{r}$. This property of mapping (13) is known as port symmetry.

In (13), $\boldsymbol{K}$ is the $(6 \times 6)$ symmetric positive semidefinite stiffness matrix. The $(3 \times 3)$ matrices $\boldsymbol{K}_{p}$ and $\boldsymbol{K}_{o}$, called, respectively, translational stiffness and rotational stiffness, are also symmetric. It can be shown that, if the $(3 \times 3)$ matrix $\boldsymbol{K}_{c}$, called coupling stiffness is symmetric, there is maximum decoupling between rotation and translation [15].

There are special cases in which $\boldsymbol{K}_{c}=\boldsymbol{O}_{3}$ and no coupling exists between translation and rotation, i.e., a relative translation of the bodies results in a wrench corresponding to a pure force along an axis through the center of stiffness; also, a relative rotation of the bodies results in a wrench that is equivalent to a pure torque about an axis through the center of stiffness. In these cases, the center of stiffness and compliance coincides. Mechanical systems with completely decoupled behavior are, e.g., the remote center of compliance (RCC) devices. In this paper, only the case in which $\boldsymbol{K}_{c}=\boldsymbol{O}_{3}$ is considered.

Since $\boldsymbol{K}_{p}$ is symmetric, there exists a rotation matrix $\boldsymbol{R}_{p}$ with respect to frame $\Sigma_{d}=\Sigma_{r}$, at equilibrium, such that $\boldsymbol{K}_{p}=\boldsymbol{R}_{p} \boldsymbol{\Gamma}_{p} \boldsymbol{R}_{p}^{T}$, and $\boldsymbol{\Gamma}_{p}$ is a diagonal matrix whose diagonal elements are the principal translational stiffnessess in the directions corresponding to the columns of rotation matrix $\boldsymbol{R}_{p}$, known as principal axes of translational stiffness. Analogously, $\boldsymbol{K}_{o}$ can be expressed as $\boldsymbol{K}_{o}=\boldsymbol{R}_{o} \boldsymbol{\Gamma}_{o} \boldsymbol{R}_{o}^{T}$, where the diagonal elements of $\boldsymbol{\Gamma}_{o}$ are the principal rotational stiffnesses about the axes corresponding to the columns of rotation matrix $\boldsymbol{R}_{o}$, known as the principal axes of rotational stiffness. In sum, a $(6 \times 6)$ stiffness matrix can be specified, with respect to a frame with the origin in the center of stiffness, in terms of the principal stiffness parameters and principal axes [16].

Note that the mechanical stiffness defined by (13) describes the behavior of an ideal 6-DOF spring that stores potential energy. The potential energy function of an ideal stiffness depends 
only on the relative position and orientation of the two attached bodies and is port symmetric.

\section{B. Geometrically Consistent Stiffness}

To achieve a geometrically consistent 6-DOF stiffness, the properties of the ideal mechanical stiffness for small displacements should be extended to the case of finite displacements. Moreover, to guarantee asymptotic stability in the sense of Lyapunov, a suitable potential elastic energy function must be defined.

Hereafter, the case in which the coupling stiffness matrix is zero will be considered, since it is the most common in practical applications. Hence, the potential elastic energy can be computed as the sum of a translational potential energy and a rotational potential energy.

The translational potential energy can be defined as

$$
V_{p}=\frac{1}{2} \boldsymbol{\Delta} \boldsymbol{p}_{d r}^{T} \boldsymbol{K}_{p}^{\prime} \boldsymbol{\Delta} \boldsymbol{p}_{d r}
$$

with

$$
\boldsymbol{K}_{p}^{\prime}=\frac{1}{2} \boldsymbol{R}_{d} \boldsymbol{K}_{p} \boldsymbol{R}_{d}^{T}+\frac{1}{2} \boldsymbol{R}_{r} \boldsymbol{K}_{p} \boldsymbol{R}_{r}^{T}
$$

where $\boldsymbol{K}_{p}$ is a $(3 \times 3)$ symmetric and positive-definite matrix. The use of $\boldsymbol{K}_{p}^{\prime}$ in lieu of $\boldsymbol{K}_{p}$ in (14) guarantees that the potential energy is port symmetric also in the case of finite displacements. Matrices $\boldsymbol{K}_{p}^{\prime}$ and $\boldsymbol{K}_{p}$ coincide at equilibrium (i.e., when $\boldsymbol{R}_{d}=$ $\boldsymbol{R}_{r}$ ) and in the case of isotropic translational stiffness (i.e., when $\left.\boldsymbol{K}_{p}=k_{p} \boldsymbol{I}\right)$.

The computation of the power $\dot{V}_{p}$ yields

$$
\dot{V}_{p}=\boldsymbol{\Delta} \dot{\boldsymbol{p}}_{d r}^{r T} \boldsymbol{f}_{\Delta p}^{r}+\boldsymbol{\Delta} \boldsymbol{\omega}_{d r}^{r T} \boldsymbol{\mu}_{\Delta p}^{r}
$$

where $\boldsymbol{\Delta} \dot{\boldsymbol{p}}_{d r}^{r}$ is the time derivative of the position displacement $\boldsymbol{\Delta} \boldsymbol{p}_{d r}^{r}=\boldsymbol{R}_{r}^{\mathrm{T}}\left(\boldsymbol{p}_{d}-\boldsymbol{p}_{r}\right)$, while $\boldsymbol{\Delta} \boldsymbol{\omega}_{d r}^{r}=\boldsymbol{R}_{r}^{\mathrm{T}}\left(\boldsymbol{\omega}_{d}-\boldsymbol{\omega}_{r}\right)$. Vectors $\boldsymbol{f}_{\Delta p}^{r}$ and $\boldsymbol{\mu}_{\Delta p}^{r}$ are, respectively, the elastic force and moment applied to the end effector in the presence of the finite position displacement $\boldsymbol{\Delta} \boldsymbol{p}_{d r}^{r}$. These vectors have the following expressions when computed in the base frame:

$$
\boldsymbol{f}_{\Delta p}=\boldsymbol{K}_{p}^{\prime} \boldsymbol{\Delta} \boldsymbol{p}_{d r}, \quad \boldsymbol{\mu}_{\Delta p}=\boldsymbol{K}_{p}^{\prime \prime} \boldsymbol{\Delta} \boldsymbol{p}_{d r}
$$

with

$$
\boldsymbol{K}_{p}^{\prime \prime}=\frac{1}{2} \boldsymbol{S}\left(\boldsymbol{\Delta} \boldsymbol{p}_{d r}\right) \boldsymbol{R}_{d} \boldsymbol{K}_{p} \boldsymbol{R}_{d}^{T}
$$

Vector $\boldsymbol{h}_{\Delta p}=\left[\begin{array}{ll}\boldsymbol{f}_{\Delta p}^{T} & \boldsymbol{\mu}_{\Delta p}^{T}\end{array}\right]^{T}$ is the elastic wrench applied to frame $\Sigma_{r}$ in the presence of a finite position displacement $\boldsymbol{\Delta} \boldsymbol{p}_{d r}$ and a null orientation displacement. The moment $\boldsymbol{\mu}_{\Delta p}$ is null in the case of isotropic translational stiffness.

Using the vector part of the unit quaternion $\mathcal{Q}_{d r}$, the orientation potential energy has the form

$$
V_{o}=2 \boldsymbol{\epsilon}_{d r}^{r T} \boldsymbol{K}_{o} \boldsymbol{\epsilon}_{d r}^{r}
$$

where $\boldsymbol{K}_{o}$ is a $(3 \times 3)$ symmetric and positive-definite matrix. Function $V_{o}$ is port symmetric because $\epsilon_{d r}^{r}=-\epsilon_{r d}^{d}$.

The computation of the power $\dot{V}_{o}$ yields

$$
\dot{V}_{o}=\boldsymbol{\Delta} \boldsymbol{\omega}_{d r}^{r T} \boldsymbol{\mu}_{\Delta o}^{r}
$$

where

$$
\boldsymbol{\mu}_{\Delta o}=\boldsymbol{K}_{o}^{\prime} \boldsymbol{\epsilon}_{d r}
$$

with

$$
\boldsymbol{K}_{o}^{\prime}=2 \boldsymbol{E}^{T}\left(\eta_{d r}, \boldsymbol{\epsilon}_{d r}\right) \boldsymbol{R}_{r} \boldsymbol{K}_{o} \boldsymbol{R}_{r}^{T}
$$

as $\boldsymbol{E}\left(\eta_{d r}, \boldsymbol{\epsilon}_{d r}\right)=\eta_{d r} \boldsymbol{I}-\boldsymbol{S}\left(\boldsymbol{\epsilon}_{d r}\right)$. The earlier equations show that a finite orientation displacement $\boldsymbol{\epsilon}_{d r}=\boldsymbol{R}_{r}^{T} \boldsymbol{\epsilon}_{d r}^{r}$ produces an elastic wrench $\boldsymbol{h}_{\Delta o}=\left[\begin{array}{ll}\mathbf{0}^{T} & \boldsymbol{\mu}_{\Delta o}^{T}\end{array}\right]^{T}$ that is equivalent to a pure moment.

Hence, the total elastic wrench in the presence of a finite position displacement and orientation displacement of the desired frame $\Sigma_{d}$ with respect to the end-effector frame $\Sigma_{r}$ can be defined in the base frame as

$$
\boldsymbol{h}_{\Delta}=\boldsymbol{h}_{\Delta p}+\boldsymbol{h}_{\Delta o}
$$

where $\boldsymbol{h}_{\Delta p}$ and $\boldsymbol{h}_{\Delta o}$ are computed according to (15) and (17), respectively.

Using (18) for the computation of the elastic wrench in the case of an infinitesimal twist displacement $\boldsymbol{\delta} \boldsymbol{x}_{d r}^{r}$ near the equilibrium, and discarding the high-order infinitesimal terms, yields the linear mapping

$$
\boldsymbol{h}_{r}^{r}=\boldsymbol{K} \boldsymbol{\delta} \boldsymbol{x}_{d r}^{r}=\left[\begin{array}{cc}
\boldsymbol{K}_{p} & \boldsymbol{O} \\
\boldsymbol{O} & \boldsymbol{K}_{o}
\end{array}\right] \boldsymbol{\delta} \boldsymbol{x}_{d r}^{r} .
$$

Therefore, $\boldsymbol{K}$ represents the stiffness matrix of an ideal spring with respect to a frame $\Sigma_{r}$ (coinciding with $\Sigma_{d}$ at equilibrium) with the origin in the center of stiffness. Moreover, it can be shown that, using definition (18), the physical/geometrical meaning of the principal stiffnesses and of the principal axes for matrices $\boldsymbol{K}_{p}$ and $\boldsymbol{K}_{o}$ are preserved also in the case of large displacements.

The earlier results imply that the active stiffness matrix $\boldsymbol{K}$ can be set in a geometrically consistent way with respect to the task at hand.

\section{Six-DOF Impedance}

The equation that defines the dynamic behavior of a generalized mechanical impedance is

$$
\boldsymbol{M} \boldsymbol{\Delta} \dot{\boldsymbol{v}}_{d r}^{r}+\boldsymbol{D} \boldsymbol{\Delta} \boldsymbol{v}_{d r}^{r}+\boldsymbol{h}_{\Delta}^{r}=\boldsymbol{h}_{r}^{r}
$$

where $\boldsymbol{M}$ and $\boldsymbol{D}$ are $(6 \times 6)$ symmetric and positive-definite matrices, $\boldsymbol{\Delta} \dot{\boldsymbol{v}}_{d r}^{r}=\dot{\boldsymbol{v}}_{d}^{r}-\dot{\boldsymbol{v}}_{r}^{r}, \boldsymbol{\Delta} \boldsymbol{v}_{d r}^{r}=\boldsymbol{v}_{d}^{r}-\boldsymbol{v}_{r}^{r}, \dot{\boldsymbol{v}}_{d}^{r}$ and $\boldsymbol{v}_{d}^{r}$ are, respectively, the acceleration and the velocity of the desired frame $\Sigma_{d}, \boldsymbol{h}_{\Delta}^{r}$ is the elastic wrench expressed as in (18), and $\boldsymbol{h}_{r}^{r}$ is the external wrench; all the earlier quantities are referred to $\boldsymbol{\Sigma}_{r}$.

The asymptotic stability of the equilibrium, in the case $\boldsymbol{h}_{r}^{r}=$ 0, can be proven by considering the Lyapunov function

$$
V=\frac{1}{2} \boldsymbol{\Delta} \boldsymbol{v}_{d r}^{r T} \boldsymbol{K} \boldsymbol{\Delta} \boldsymbol{v}_{d r}^{r}+V_{p}+V_{o}
$$

where $V_{p}$ and $V_{o}$ are defined in (14) and (16), respectively, whose time derivative along the trajectories of system (20) is the negative semidefinite function

$$
\dot{V}=-\boldsymbol{\Delta} \boldsymbol{v}_{d r}^{r T} \boldsymbol{D} \boldsymbol{\Delta} \boldsymbol{v}_{d r}^{r} .
$$


When $\boldsymbol{h}_{r}^{r} \neq \mathbf{0}$, a different asymptotically stable equilibrium can be found, corresponding to a nonnull displacement of the desired frame $\Sigma_{d}$ with respect to $\Sigma_{r}$. The new equilibrium is the solution of the equation $\boldsymbol{h}_{\Delta}^{r}=\boldsymbol{h}_{r}^{r}$.

When $\Sigma_{d}$ is constant, (20) has the meaning of a true 6-DOF mechanical impedance if $\boldsymbol{M}$ is chosen as

$$
\boldsymbol{M}=\left[\begin{array}{cc}
m_{p} \boldsymbol{I} & \boldsymbol{O} \\
\boldsymbol{O} & \boldsymbol{J}_{o}
\end{array}\right]
$$

where $m_{p}$ is a mass, $\boldsymbol{J}_{o}$ is a $(3 \times 3)$ inertia tensor, and $\boldsymbol{D}$ is chosen as a block diagonal matrix with $(3 \times 3)$ blocks. The physically equivalent system is a body of mass $m_{p}$, inertia tensor $\boldsymbol{J}_{o}$ with respect to frame $\Sigma_{r}$, subject to an external wrench $\boldsymbol{h}_{r}^{r}$. This frame is virtually connected to frame $\Sigma_{d}$ through a 6DOF ideal spring with stiffness matrix $K$ and is subject to viscous forces and moments with damping $\boldsymbol{D}$. Function $V$ in (21) represents the total energy of the body, which is the sum kinetic energy and of the potential elastic energy.

In the absence of interaction, the impedance guarantees that $\Sigma_{r}$ asymptotically follows the desired frame $\Sigma_{d}$. In the presence of contact with the environment, a compliant dynamic behavior is imposed on the end effector, according to impedance (20), and the contact wrench is kept bounded at the expense of a finite position displacement and orientation displacement between $\Sigma_{d}$ and $\Sigma_{r}$.

\section{IMPEDANCE CONTROL FOR COOPERATIVE MANIPULATORS}

\section{A. External Impedance}

In order to achieve bounded contact forces exchanged with the environment, an impedance-based strategy can be pursued, as in [17].

Let $\mathcal{T}_{e_{d}}$ be the set of motion variables describing the assigned trajectory for the object-desired frame $\Sigma_{e_{d}}$. Then, the corresponding set of reference variables $\mathcal{T}_{e_{r}}=\left\{\boldsymbol{p}_{e_{r}}, \mathcal{Q}_{e_{r}}\right.$, $\left.\dot{\boldsymbol{p}}_{e_{r}}, \boldsymbol{\omega}_{e_{r}}, \ddot{\boldsymbol{p}}_{e_{r}}, \dot{\boldsymbol{\omega}}_{e_{r}}\right\}$ can be generated by integrating the differential equation

$$
\begin{aligned}
& (\boldsymbol{A}-\boldsymbol{I}) \boldsymbol{M}_{e} \dot{\boldsymbol{v}}_{e_{r}}^{e_{r}}+\boldsymbol{D}_{e} \boldsymbol{v}_{e_{r}}^{e_{r}}+\boldsymbol{h}_{\Delta, e}^{e_{r}}= \\
& \quad-\boldsymbol{h}_{e}^{e_{r}}-\boldsymbol{C}\left(\boldsymbol{v}_{e}^{e_{r}}\right) \boldsymbol{v}_{e}^{e_{r}}-\boldsymbol{g}_{e}^{e_{r}}+\boldsymbol{A} \boldsymbol{M}_{e} \dot{\boldsymbol{v}}_{e_{d}}^{e_{r}}+\boldsymbol{D}_{e} \boldsymbol{v}_{e_{d}}^{e_{r}}
\end{aligned}
$$

where $M_{e}$ is the object mass matrix

$$
\begin{aligned}
\boldsymbol{A} & =\left[\begin{array}{cc}
\alpha_{p} \boldsymbol{I} & \boldsymbol{O} \\
\boldsymbol{O} & \alpha_{o} \boldsymbol{I}
\end{array}\right] \\
\boldsymbol{D}_{e} & =\left[\begin{array}{cc}
\boldsymbol{D}_{p e} & \boldsymbol{O} \\
\boldsymbol{O} & \boldsymbol{D}_{o e}
\end{array}\right]
\end{aligned}
$$

and, according to (15) and (17), the elastic wrench is given by

$$
\boldsymbol{h}_{\Delta, e}=\boldsymbol{h}_{\Delta, e p}+\boldsymbol{h}_{\Delta, e o}=\left[\begin{array}{c}
\boldsymbol{K}_{p e}^{\prime} \Delta \boldsymbol{p}_{e_{d} e_{r}} \\
\boldsymbol{K}_{p e}^{\prime \prime} \Delta \boldsymbol{p}_{e_{d} e_{r}}+\boldsymbol{K}_{o e}^{\prime} \boldsymbol{\epsilon}_{e_{d} e_{r}}
\end{array}\right] \text {. }
$$

It is straightforward to prove that asymptotic stability of (22) is ensured if the scalars $\alpha_{p}$ and $\alpha_{o}$ in the matrix $\boldsymbol{A}$ are greater than or equal to 1 . Noticeably, in order to compute (22), only end-effector forces and moments need to be measured (e.g., via wrist-mounted force/torque sensors), since $\boldsymbol{h}_{e}^{e_{r}}=\boldsymbol{R}_{e_{r}}^{T} \boldsymbol{h}_{e}$, and $\boldsymbol{h}_{e}$ can be readily computed via (3) by using only $\boldsymbol{h}$.
The earlier choice of the object reference trajectory enforces an impedance behavior with inertia $\boldsymbol{A} \boldsymbol{M}_{e}$, damping $\boldsymbol{D}_{e}$, and stiffness

$$
\boldsymbol{K}_{e}=\left[\begin{array}{cc}
\boldsymbol{K}_{p e} & \boldsymbol{O} \\
\boldsymbol{O} & \boldsymbol{K}_{o e}
\end{array}\right] .
$$

In fact, assuming perfect tracking, the frames $\Sigma_{e}$ and $\Sigma_{e_{r}}$ can be assumed to be aligned. Hence, folding (22) into the object dynamic equation (2), the following impedance equation is obtained:

$$
\boldsymbol{A} \boldsymbol{M}_{e} \boldsymbol{\Delta} \dot{\boldsymbol{v}}_{e_{d} e_{r}}^{e_{r}}+\boldsymbol{D}_{e} \boldsymbol{\Delta} \boldsymbol{v}_{e_{d} e_{r}}^{e_{r}}+\boldsymbol{h}_{\Delta, e}^{e_{r}}=\boldsymbol{h}_{\mathrm{env}}^{e_{r}}
$$

where all the displacements are formally equal to those in (20).

It is worth noticing that, differently from [17], direct measurement of the object acceleration is not required due to the feedforward terms in (22) compensating for the object's dynamics. On the other hand, the mass and inertia matrices for the impedance cannot be assigned by the user in an arbitrary way, but they must be set proportional to the mass and inertia, respectively. This is due to the fact that direct measurements of the contact force $\boldsymbol{h}_{\text {env }}^{e_{r}}$ or, equivalently, of the object accelerations are not available. It is also advisable to choose the scaling factors close to the unity so as to avoid large modifications of the system's mass/inertia characteristics.

Finally, the corresponding reference trajectories for the inner loops $\mathcal{T}_{k_{d}}=\left\{\boldsymbol{p}_{k_{d}}, \mathcal{Q}_{k_{d}}, \dot{\boldsymbol{p}}_{k_{d}}, \boldsymbol{\omega}_{k_{d}}, \ddot{\boldsymbol{p}}_{k_{d}}, \dot{\boldsymbol{\omega}}_{k_{d}}\right\}\left(k_{d}=1,2\right)$ are then computed from $\mathcal{T}_{e_{r}}$ according to the model of the grasp geometry (7).

\section{B. Internal Impedance}

The impedance paradigm can be exploited to achieve bounded internal forces as well; with this purpose, a similar strategy, as in [18], can be adopted. Namely, an impedance behavior is imposed between the position/orientation displacements at each end effector and the force and moment contributions $\boldsymbol{f}_{I k}$ and $\boldsymbol{\mu}_{I k}$ in (6), i.e., for $k=1,2$

$$
\boldsymbol{M}_{I k} \Delta \dot{\boldsymbol{v}}_{k_{d} k_{r}}^{k_{r}}+\boldsymbol{D}_{I k} \Delta \boldsymbol{v}_{k_{d} k_{r}}^{k_{r}}+\boldsymbol{h}_{\Delta, I k}^{k_{r}}=\boldsymbol{h}_{I k}^{k_{r}}
$$

where

$$
\begin{aligned}
\boldsymbol{M}_{I k} & =\left[\begin{array}{cc}
m_{I k} \boldsymbol{I} & \boldsymbol{O} \\
\boldsymbol{O} & \boldsymbol{J}_{I k}
\end{array}\right] \\
\boldsymbol{D}_{I k} & =\left[\begin{array}{cc}
\boldsymbol{D}_{p I k} & \boldsymbol{O} \\
\boldsymbol{O} & \boldsymbol{D}_{o I k}
\end{array}\right]
\end{aligned}
$$

and

$$
\boldsymbol{h}_{\Delta, I k}=\left[\begin{array}{c}
\boldsymbol{K}_{p I k}^{\prime} \boldsymbol{\Delta} \boldsymbol{p}_{k_{d} k_{r}} \\
\boldsymbol{K}_{p I k}^{\prime \prime} \boldsymbol{\Delta} \boldsymbol{p}_{k_{d} k_{r}}+\boldsymbol{K}_{o I k}^{\prime} \boldsymbol{\epsilon}_{k_{d} k_{r}}
\end{array}\right] .
$$

Then, the trajectory $\mathcal{T}_{k_{r}}=\left\{\boldsymbol{p}_{k_{r}}, \mathcal{Q}_{k_{r}}, \dot{\boldsymbol{p}}_{k_{r}}, \boldsymbol{\omega}_{k_{r}}, \ddot{\boldsymbol{p}}_{k_{r}}, \dot{\boldsymbol{\omega}}_{k_{r}}\right\}$ is computed by integrating (24). Noticeably, in order to compute (24), only end-effector forces and moments need to be measured (e.g., via wrist-mounted force/torque sensors), since for $k=$ $1,2, \boldsymbol{h}_{I k}^{k_{r}}=\boldsymbol{R}_{k_{r}}^{T} \boldsymbol{h}_{I k}$, and $\boldsymbol{h}_{I}$ can be readily computed via (6) by using only $\boldsymbol{h}$.

The effect of the impedance is that of adjusting the reference trajectories for the two end effectors so as to avoid building 
up of large internal forces. In fact, (22) enforces an impedance behavior with inertia $\boldsymbol{M}_{I k}$, damping $\boldsymbol{D}_{I k}$, and stiffness

$$
\boldsymbol{K}_{I k}=\left[\begin{array}{cc}
\boldsymbol{K}_{p I k} & \boldsymbol{O} \\
\boldsymbol{O} & \boldsymbol{K}_{o I k}
\end{array}\right] .
$$

Moreover, by following the guidelines in [6] and [18], it can be shown that the object motion at steady state is not affected by the impedance on the internal forces. In fact, at steady state, (24), written in the base frame, reduces to $(k=1,2)$

$$
\boldsymbol{h}_{\Delta, I k}=\boldsymbol{h}_{I k}
$$

which implies that

$$
\left[\begin{array}{l}
\boldsymbol{h}_{\Delta, I 1} \\
\boldsymbol{h}_{\Delta, I 2}
\end{array}\right]=\boldsymbol{V} \boldsymbol{V}^{\dagger} \boldsymbol{h} .
$$

In other words, the elastic contribution of the internal impedance lies in the range of $\boldsymbol{V}$, i.e., in the null space of $\boldsymbol{W}$. Hence, by virtue of (3) and (4), under the assumption of tight grasp and rigid manipulated object, such a contribution cannot influence the object's steady-state position/orientation, by definition of internal forces.

Note that, due to the uncoupling between external and internal motion variables, the activation of the internal impedance does not influence the object's desired position/orientation at steady state.

As for the implementation scheme of the two impedance equations (23) and (24), depicted in Fig. 1, a two-loop arrangement is adopted. Namely, the outer loop computes, by integrating (22), the object's reference motion variables $\mathcal{T}_{e_{r}}$ from the desired object's motion $\mathcal{T}_{e_{d}}$; the latter are transformed to desired end-effector variables $\mathcal{T}_{k_{d}}$ via the equations of the closed-chain constraints (7). Then, the internal impedance filter (24) is integrated to generate the joints references $\mathcal{T}_{k_{r}}$. Of course, this arrangement makes possible individual activation/deactivation of the two impedances. Namely, deactivation of each impedance filter can achieved by simply zeroing the corresponding force feedback.

\section{EXPERIMENTS}

The goal of the experimental study reported in this section is that of demonstrating the advantages of applying a combined internal/external impedance control scheme with respect to the adoption of a sole internal (or external) impedance. Moreover, the experiments are carried out under nonideal conditions (e.g., loose grasp and/or deformable object, slipping, backlash, and friction at the joints), quite common in industrial setups. Such effects are not taken into account in the design of the control laws, since they are difficult to model and/or would lead to extremely complex control laws. In this way, the robustness and reliability of the impedance control approach can be assessed.

The experiments have been performed on a setup composed of two industrial robots COMAU Smart-3 S with C3G 9000 control units, available in the Portfolio Risk Management (PRISMA) Laboratory. These are six-revolute-joint anthropomorphic manipulators. One of the robots is mounted on a sliding track, which has been considered mechanically braked for the purposes of this paper.

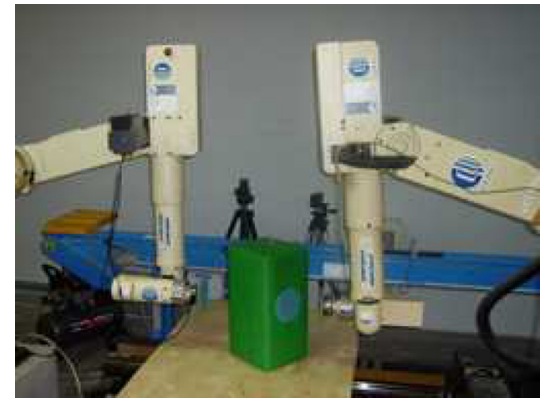

Fig. 2. Experimental setup.

The robotic system is equipped with an open controller architecture. Namely, a standard PC, with a real-time software environment, named RePLiCS [27], running under a real-time variant of the Linux operative system, can replace the original C3G 9000 controllers, thus allowing implementation of advanced control schemes for the single robots and the dual-arm robotic cell. It must be remarked that $\mathrm{C} 3 \mathrm{G} 9000$ is replaced by the $\mathrm{PC}$ in the sense that the $\mathrm{PC}$ is in charge of planning the desired motion and computing the control algorithm, while the C3G 9000 is used solely as an interface between joints sensors (resolvers at the joints shafts) and servo amplifiers (which, in turn, drive the brushless motors actuating the joints motion). Seven different operating modes are available, allowing the PC to interact with the original controller both at the trajectory generation level and at the joint control level. In the operating mode used for experiments, the PC is in charge of acquiring data from the resolvers and force/torque sensors, computing the control algorithm, and passing the references to the servo amplifiers of both the robots at 1-ms sampling time.

The sensing capabilities of the robotic cell are completed by two 6-axis force/torque sensors ATIFT30-100 with a force range of $\pm 130 \mathrm{~N}$ and a torque range of $\pm 10 \mathrm{~N} \cdot \mathrm{m}$, mounted at either arm's wrist. The sensor is connected to the PC by a parallel interface board that provides readings of six components of generalized force at $1 \mathrm{~ms}$.

In the experiments, the two manipulators grasp a $(0.24 \mathrm{~m} \times 0.24 \mathrm{~m} \times 0.45 \mathrm{~m})$ cardbox, with $m_{e}=1.3792 \mathrm{~kg}$ mass and inertia tensor $\boldsymbol{J}_{e}^{e}=\operatorname{diag}\{0.03,0.03,0.0123\} \mathrm{kg} \mathrm{m}^{2}$ (expressed in the frame $\Sigma_{e}$ located in the object's center of mass); the object's parameters have been estimated via computer-added design (CAD) modeling techniques. The grasp is achieved via two couples of rubber disks; each couple consists of two disks mounted, respectively, on the end effector of the manipulator and on one of the object's faces at a given grasp area. The environment interacting with the cooperative system is a planar wooden horizontal surface (see Fig. 2), whose position and orientation is unknown to the controller.

The assigned task has a total duration of $115 \mathrm{~s}$ and is composed of three phases.

1) In the first phase (grasping), with a duration of $9 \mathrm{~s}$, the manipulators, moving from their initial configuration, grasp the object using the two rubber disks.

2) In the second phase (object not interacting with the environment), with a duration of $30 \mathrm{~s}$, the manipulators lift the 

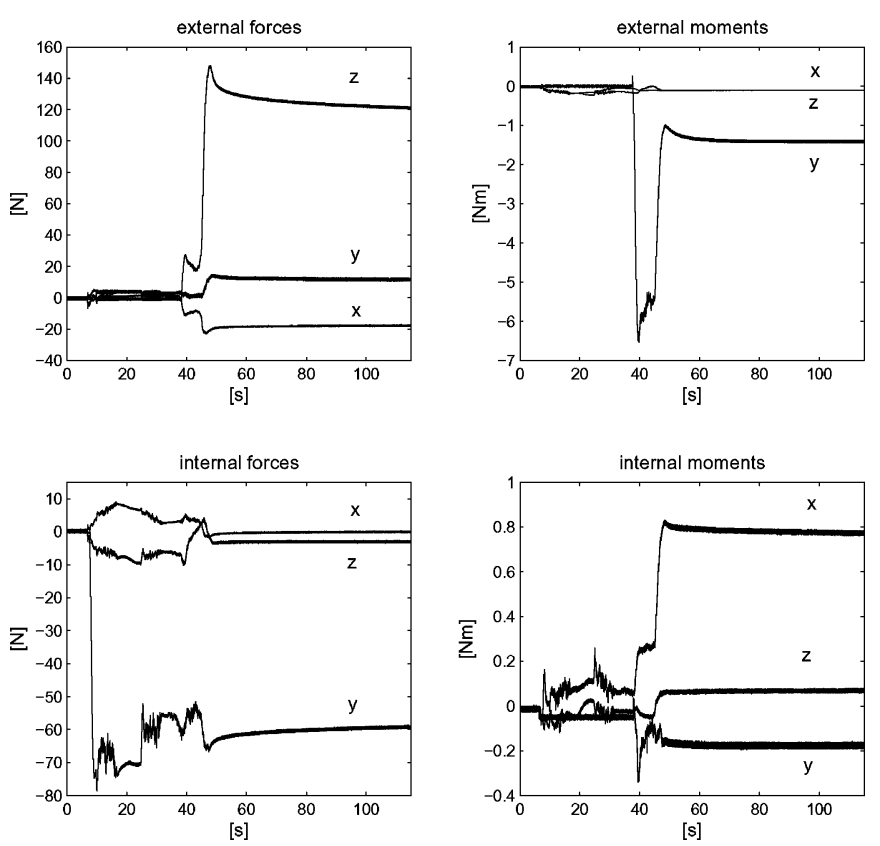

Fig. 3. First case study. Top: external forces and moments. Bottom: internal forces and moments.
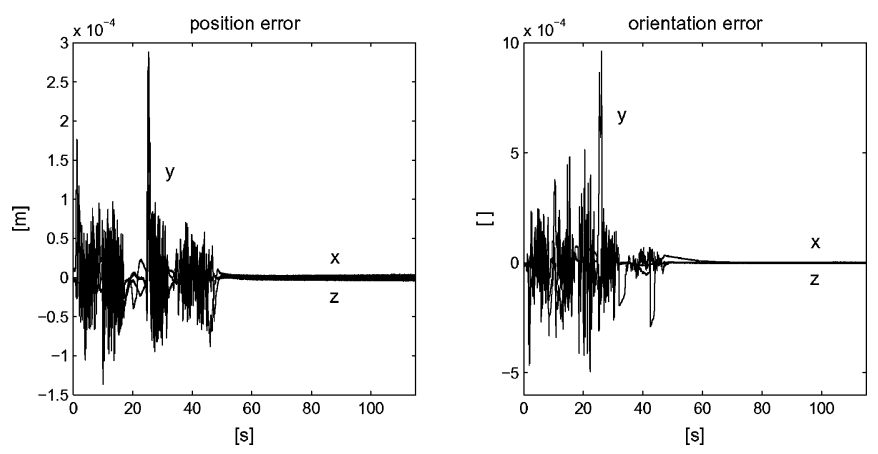

Fig. 4. First case study. Object position and orientation errors.

commonly held object and change the object orientation of an angle of $0.23 \mathrm{rad}$ about the axis between the two end-effectors positions (corresponding to the $y$-axis of the base frame), then the object is moved down so as to reach contact with the environment.

3) In the last phase (object interacting with the environment), with a duration of $76 \mathrm{~s}$, the object is commanded to follow a vertical path of $0.011 \mathrm{~m}$, with constant orientation, pushing against the environment.

The gains of the PID control law are

$$
\begin{aligned}
& \boldsymbol{G}_{P, k}=\operatorname{diag}\{77,233,60,25,17,0.4\} \\
& \boldsymbol{G}_{V, k}=\operatorname{diag}\{0.77,1.55,1,0.33,0.17,0.06\} \\
& \boldsymbol{G}_{I, k}=\operatorname{diag}\{0.24,0.73,0.18,0.077,0.11,0.0027\} .
\end{aligned}
$$

The gains of the second-order CLIK algorithm are: $k_{P_{p}}=$ $150, k_{P_{o}}=50, k_{V_{p}}=192$, and $k_{V_{o}}=13.8$.

The parameters of the impedance equation (22) have been set to $\alpha_{p}=1.5, \boldsymbol{D}_{p e}=\operatorname{diag}\{1.3,1.3,1.5\} \cdot 10^{3}, \boldsymbol{K}_{p e}=$ $\operatorname{diag}\{6.5,6.5,8\} \cdot 10^{2}, \alpha_{o}=1.5, \boldsymbol{D}_{o e}=10 \boldsymbol{I}_{3}$, and $\boldsymbol{K}_{o e}=$
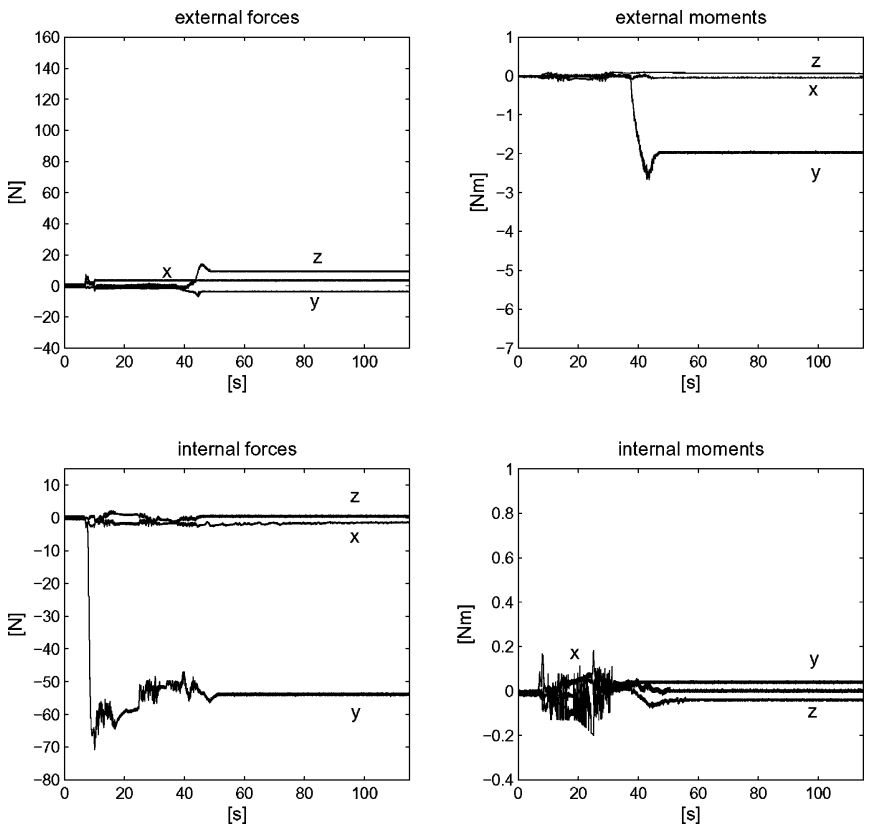

Fig. 5. Second case study. Top: external forces and moments. Bottom: internal forces and moments.
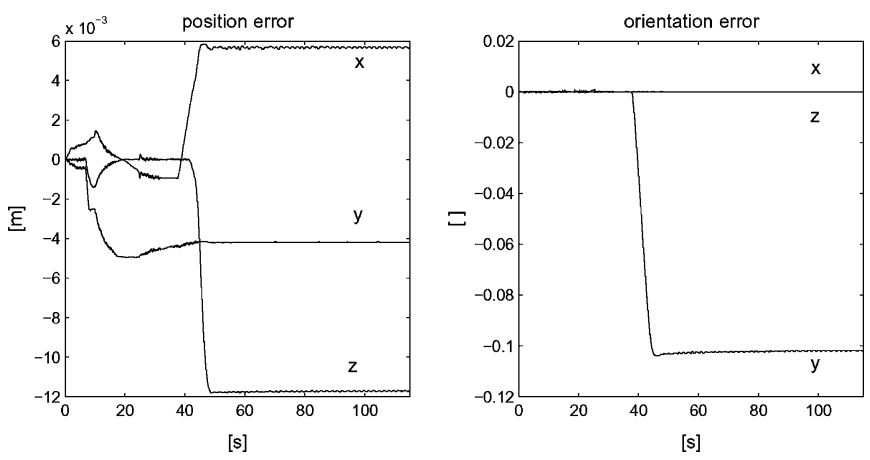

Fig. 6. Second case study. Object position and orientation errors.

$\operatorname{diag}\{1.5,1.5,10\}$. The parameters of the impedance equation (22) have been set to $\boldsymbol{M}_{p I}=\operatorname{diag}\{2,1,2\}, \boldsymbol{D}_{p I}=10^{3} \boldsymbol{I}_{3}$, $\boldsymbol{K}_{p I}=\operatorname{diag}\{3.7,5,3.7\} \cdot 10^{2}, \boldsymbol{M}_{o I}=2 \boldsymbol{I}_{3}, \boldsymbol{D}_{o I}=80 \boldsymbol{I}_{3}$, and $\boldsymbol{K}_{o I}=2 \boldsymbol{I}_{3}$. Notice that the stiffness matrices have been chosen so as to ensure a compliant behavior (limited values of contact force and moment), while the damping matrices have been chosen so as to guarantee a well-damped behavior.

\section{A. Pure Positional Control}

In the first case study, a purely positional control strategy is adopted, i.e., both the impedance controllers are deactivated and only the inner motion control loop is active. Fig. 3 shows the time histories of the external forces and moments $\boldsymbol{h}_{e}$, as well as the internal forces and moments $\boldsymbol{h}_{i}$. The position error $\boldsymbol{p}_{e_{d}}-\boldsymbol{p}_{e}$ and orientation error $\boldsymbol{\epsilon}_{e_{d} e}=\boldsymbol{R}_{e} \boldsymbol{\epsilon}_{e_{d} e}^{e}$ of the object are reported in Fig. 4. Before the contact, the main contribution to the external force is due to the gravity force acting on the object; after the contact, the external force along the $z$-axis takes large values, since the positional control strategy tries to track the 

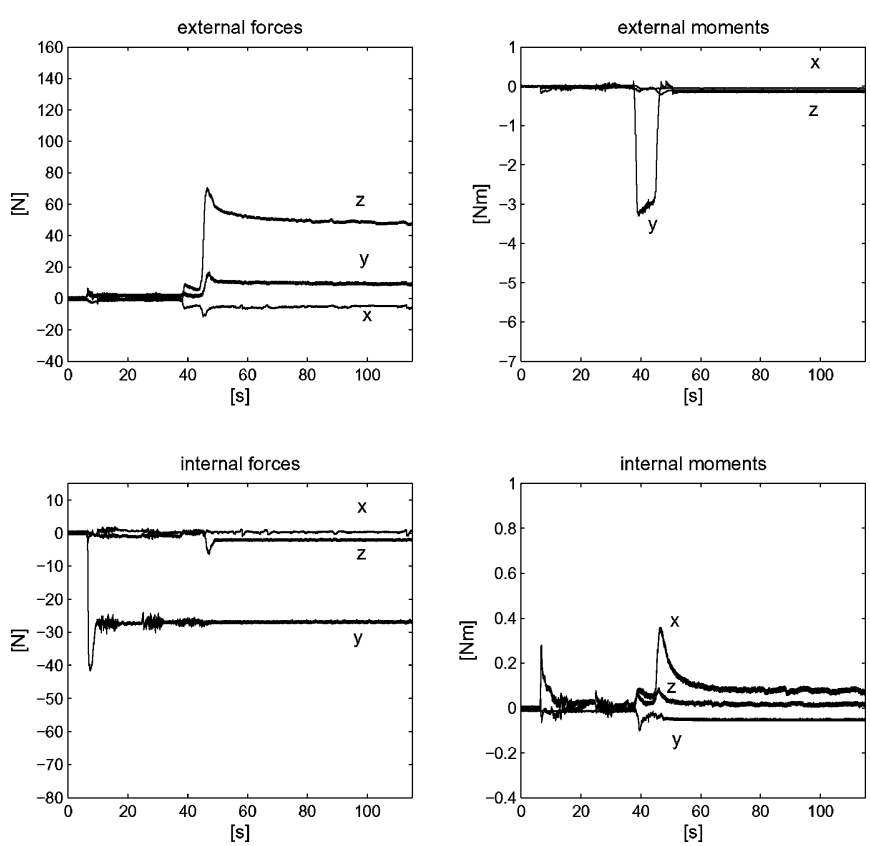

Fig. 7. Third case study. Top: external forces and moments. Bottom: internal forces and moments.

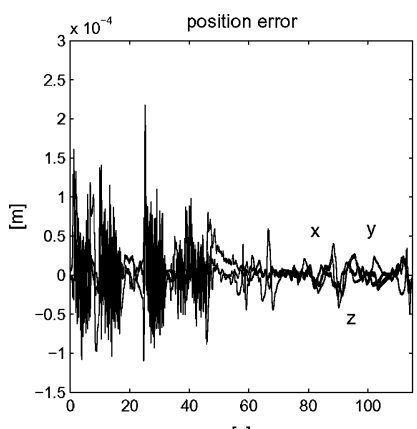

[s]

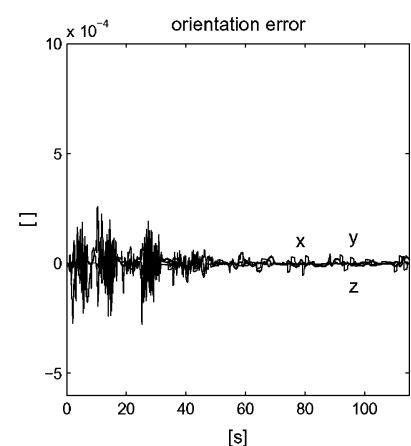

[s]
Fig. 8. Third case study. Object position and orientation errors.

commanded position and orientation trajectories, even though the path is obstructed by the environment.

The $y$ component of the external moment is initially large (in the interval $t \in[40,45] \mathrm{s}$ ) due to the misalignment about the $y$-axis of the box surface with respect to the environment surface; this causes slipping of the box, about the $y$-axis, with respect to the grasping tools that, in turn, leads to a reduction of the external moment for $t>45 \mathrm{~s}$. Note that large internal forces arise after the contact, as expected.

\section{B. External Impedance Control}

In the second case study, the object/environment interaction is managed by enforcing the impedance behavior described by (22). It can be recognized that the external forces after the contact are significantly reduced (see Fig. 5) at the expense of the position and orientation errors (see Fig. 6). Notice that the most significant component of the orientation error is that about the $y$-axis, which corresponds to a significant (although bounded) external moment about the same axis. Moreover, the internal
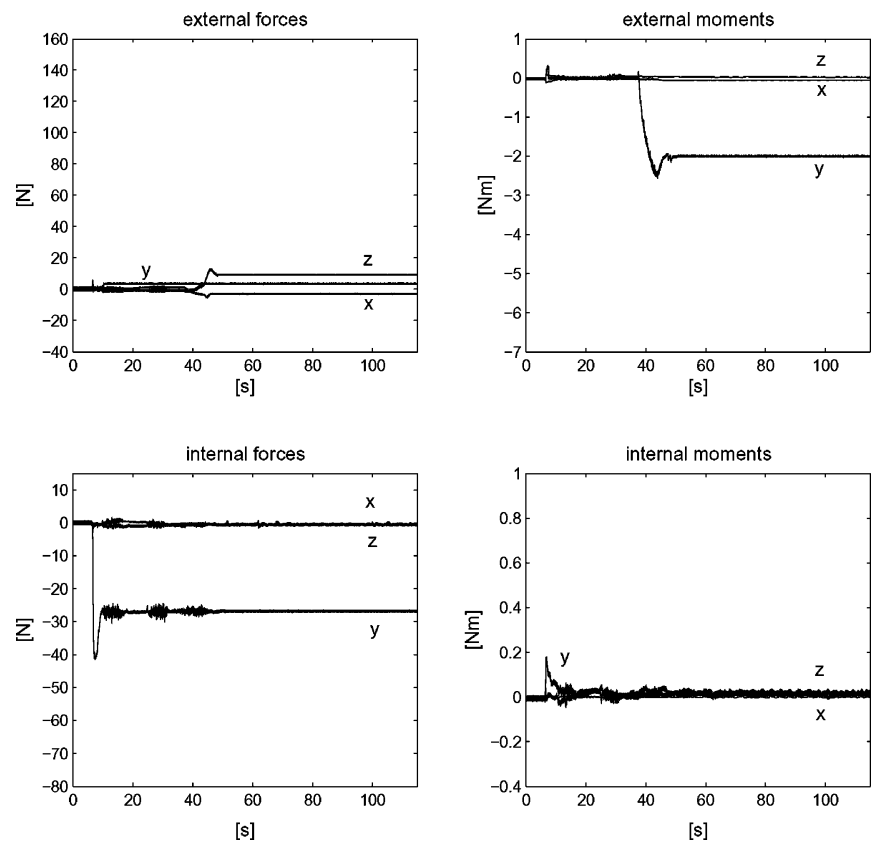

Fig. 9. Fourth case study. Top: external forces and moments. Bottom: internal forces and moments.
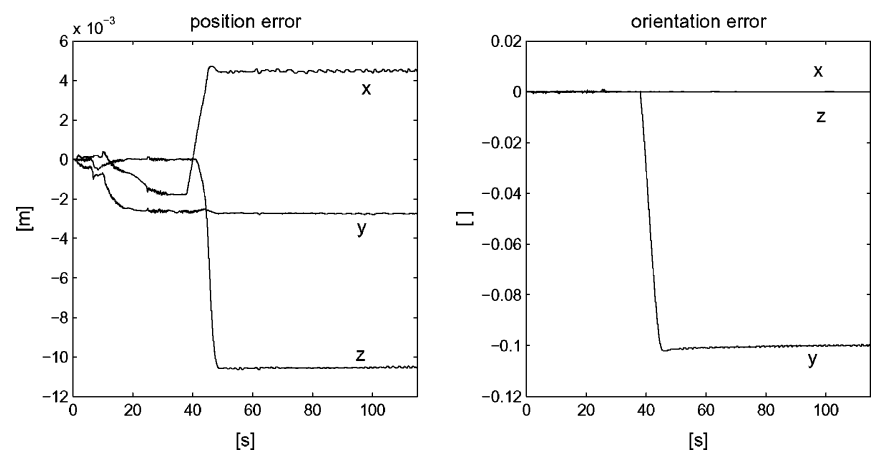

Fig. 10. Fourth case study. Object position and orientation errors.

moments (mainly due to the interaction with the environment) become smaller, while the internal force along the $y$-axis, mainly due to object squeezing, is still large. Noticeably, differently from the previous case study, slipping does not occur because the external moment about the $y$-axis, causing slipping, is limited.

\section{Internal Impedance Control}

In the third case study, only the internal impedance described by (24) is adopted. It can be recognized that a significant reduction of the internal force along the $y$-axis is obtained (see Fig. 7), and thus object squeezing is reduced. As a consequence, slipping of the box occurs about the $y$-axis, caused by the initially high external moment about the same axis due to the absence of the external impedance (see the results of the first case study). For the same reason, slipping occurs also along the $z$-axis, and this leads to lower external forces with respect to those obtained in the first case study. Also, by comparing the results in Fig. 8 with those in Fig. 6 (obtained in the absence of internal impedance), it can be noticed that position and orientation errors decrease when the internal impedance is activated. This is mainly due to 
the absence of the external impedance, which makes the object's behavior during the interaction not compliant: on the other hand, the object tracks more accurately the desired position, pushing the environment surface, thus leading to larger external forces (see Fig. 7). However, it must be remarked that such errors are computed from the end-effector positions and orientations, and thus they do not reflect the real position and orientation of the object because of slipping.

\section{External/Internal Impedance Control}

In the fourth case study, both the impedances (22) and (24) are activated, and thus the overall control scheme in Fig. 1 is considered. Note that, in this case, the external forces and moments are practically the same as in the second case study, where only the external impedance is used, as well as the position and orientation errors (Figs. 9 and 10). Moreover, the internal forces and moments are reduced, as in the third case study, where only the internal impedance is adopted. Noticeably, no slipping occurs due to the reduction of the external forces and moments. Finally, it is worth noting that object's tracking errors and external wrenches behave as in the second case study (Figs. 5 and 6). A similar result applies for the internal wrenches. This shows that external and internal impedance behaviors are practically decoupled, as predicted by theory, although small coupling effects can be observed (mainly due to object elasticity and nonrigid grasp).

\section{CONCLUSION}

In this paper, a geometrically consistent 6-DOF impedance control for dual-arm cooperative manipulators is proposed. Moreover, an experimental comparison of impedance control schemes has been presented. The results show that the adoption of the sole external impedance allows to manage the interaction of the carried object with the environment, but cannot avoid building up of large internal stresses at the object. On the other hand, the adoption of a sole internal impedance reduces the internal forces and moments at the expense of possible slipping. Finally, the adoption of both external and internal impedances achieves reduction of both external and internal forces and makes less likely the occurrence of slipping phenomena. In conclusion, validity of the geometrically consistent formulation is confirmed; moreover, the combined external/internal impedance control scheme has been revealed to be the most reliable approach to safely and effectively manage the interaction in a cooperative manipulation system.

\section{ACKNOWLEDGMENT}

The authors are solely responsible for its content. It does not represent the opinion of the European Community and the Community is not responsible for any use that might be made of the information contained therein.

\section{REFERENCES}

[1] P. Chiacchio and S. Chiaverini, Eds., Complex Robotic Systems. London, U.K.: Springer-Verlag, 1998.
[2] M. Uchiyama and P. Dauchez, "Symmetric kinematic formulation and non-master/slave coordinated control of two-arm robots," Adv. Robot., vol. 7, pp. 361-383, 1993.

[3] J. T. Wen and K. Kreutz, "Motion and force control of multiple robotic manipulators," Automatica, vol. 28, pp. 729-743, 1992.

[4] J.-H. Jean and L.-C. Fu, "An adaptive control scheme for coordinated multimanipulator systems," IEEE Trans. Robot. Autom., vol. 9, no. 2, pp. 226-231, Apr. 1993.

[5] Y.-H. Liu and S. Arimoto, "Decentralized adaptive and nonadaptive position/force controllers for redundant manipulators in cooperation," Int. J. Robot. Res., vol. 17, pp. 232-247, 1998.

[6] F. Caccavale, P. Chiacchio, and S. Chiaverini, "Task-space regulation of cooperative manipulators," Automatica, vol. 36, pp. 879-887, 2000.

[7] D. Sun and J. K. Mills, "Adaptive synchronized control for coordination of multirobot assembly tasks," IEEE Trans. Robot. Autom., vol. 18, no. 4, pp. 498-510, Aug. 2002.

[8] A. Rodriguez-Angeles and H. Nijmeijer, "Mutual synchronization of robots via estimated state feedback: A cooperative approach," IEEE Trans. Control Syst. Technol., vol. 12, no. 4, pp. 542-554, Jul. 2004.

[9] K.-Y. Lian, C.-S. Chiu, and P. Liu, "Semi-decentralized adaptive fuzzy control for cooperative multirobot systems with $H_{\infty}$ motion/internal force tracking performance," IEEE Trans. Syst., Man, Cybern. B, Cybern., vol. 32, no. 3, pp. 269-280, Jun. 2002.

[10] W. Gueaieb, F. Karray, and S. Al-Sharhan, "A robust adaptive fuzzy position/force control scheme for cooperative manipulators," IEEE Trans. Control Syst. Technol., vol. 11, no. 4, pp. 516-528, Jul. 2003.

[11] W. Gueaieb, F. Karray, and S. Al-Sharhan, "A robust hybrid intelligent position/force control scheme for cooperative manipulators," IEEE/ASME Trans. Mechatronics, vol. 12, no. 2, pp. 109-125, Apr. 2007.

[12] J. Gudio-Lau, M. A. Arteaga, L. A. Muoz, and V. Parra-Vega, "On the Control of cooperative robots without velocity measurements," IEEE Trans. control Syst. Technol., vol. 12, no. 4, pp. 600-608, Jul. 2004.

[13] C. P. Tang, R. M. Bhatt, M. Abou-Samah, and V. Krovi, "Screw-theoretic analysis framework for cooperative payload transport by mobile manipulator collectives," IIEEE/ASME Trans. Mechatronics, vol. 11, no. 2, pp. $169-168$, Apr. 2006

[14] N. Hogan, "Impedance control: An approach to manipulation: Parts I-III," ASME J. Dyn. Syst., Meas., Control., vol. 107, pp. 1-24, 1985.

[15] J. Lončarić, "Normal forms of stiffness and compliance matrices," IEEE J. Robot. Autom., vol. 3, no. 6, pp. 567-572, Dec. 1987.

[16] E. D. Fasse and J. F. Broenink, "A spatial impedance controller for robotic manipulation," IEEE Trans. Robot. Autom., vol. 13, no. 4, pp. 546-556, Aug. 1997.

[17] S. A. Schneider and R. H. Cannon, Jr., "Object impedance control for cooperative manipulation: Theory and experimental results," IEEE Trans. Robot. Autom., vol. 8, no. 3, pp. 383-394, Jun. 1992.

[18] R. G. Bonitz and T. C. Hsia, "Internal force-based impedance control for cooperating manipulators," IEEE Trans. Robot. Autom., vol. 12, no. 1, pp. 78-89, Feb. 1996.

[19] F. Caccavale and L. Villani, "Impedance control of cooperative manipulators," Mach. Intell. Robot. Control, vol. 2, pp. 51-57, 1999.

[20] F. Caccavale, C. Natale, B. Siciliano, and L. Villani, "Six-DOF impedance control based on angle/axis representations," IEEE Trans. Robot. Autom., vol. 15, no. 2, pp. 289-300, Apr. 1999.

[21] B. M. Braun, G. P. Starr, J. E. Wood, and R. Lumia, "A framework for implementing cooperative motion on industrial controllers," IEEE Trans. Robot. Autom., vol. 20, no. 3, pp. 583-589, Jun. 2004.

[22] R. E. Roberson and R. Schwertassek, Dynamics of Multibody Systems. Berlin, Germany: Springer-Verlag, 1988.

[23] J. S. C. Yuan, "Closed-loop manipulator control using quaternion feedback," IEEE Trans. Robot. Autom., vol. 4, no. 4, pp. 434-440, Aug. 1988.

[24] I. D. Walker, R. A. Freeman, and S. I. Marcus, "Analysis of motion and internal loading of objects grasped by multiple cooperating manipulators," Int. J. Robot. Res., vol. 10, pp. 396-409, 1989.

[25] F. Caccavale, S. Chiaverini, and B. Siciliano, "Second-order kinematic control of robot manipulators with Jacobian damped least-squares inverse: Theory and experiments," IEEE/ASME Trans. Mechatronics, vol. 2, no. 3, pp. 188-194, Sep. 1997.

[26] P. Rocco, "Stability of PID control for industrial robot arms," IEEE Trans. Robot. Autom., vol. 12, no. 4, pp. 606-614, Aug. 1996.

[27] F. Caccavale, V. Lippiello, B. Siciliano, and L. Villani, "RePLiCS: An environment for open real-time control of a dual-arm industrial robotic cell based on RTAI-Linux," presented at the 2005 IEEE/RSJ Int. Conf Intell. Robots Syst., Edmonton, AB, Canada, Aug. 2005. 


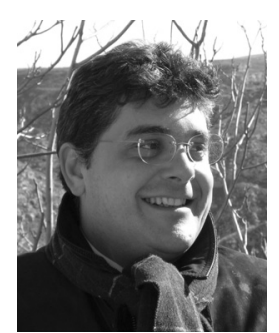

Fabrizio Caccavale (M'07-SM'08) was born in Naples, Italy, on November 14, 1965. He received the Laurea and Research Doctorate degrees in electronic engineering from the University of Naples, Naples, in 1993 and 1997, respectively.

From 1999 to 2001, he was an Assistant Professor in the Department of Computer and Systems Engineering, University of Naples. He is currently an Associate Professor in the Department of Environmental Engineering and Physics, University of Basilicata, Potenza, Italy. His current research interests include manipulator inverse kinematics techniques, cooperative robot manipulation, fault diagnosis, and nonlinear control of mechanical systems. He has authored or coauthored more than 90 journal and conference papers. He is a coauthor of the book Fondamenti di Sistemi Dinamici (McGraw-Hill, 2003, in Italian) and a coeditor of the book Fault Diagnosis for Mechatronic Systems: Recent Advances (Springer-Verlag, 2002). He is an Associate Editor of the journal Robotica.

Dr. Caccavale is an Associate Editor of the IEEE TRANSACTIONS ON CONTROL SYSTEMS TECHNOLOGY. He has been a member of the program committees of several international conferences and workshops.

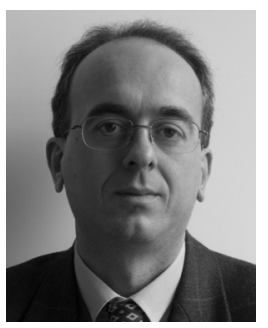

Pasquale Chiacchio was born in Naples, Italy, on September 7, 1963. He received the Laurea and Research Doctorate degrees in electronics engineering from the University of Naples, Naples, in 1987 and 1992, respectively.

He is currently a Full Professor of automatic control in the Engineering Faculty, University of Salerno, Salerno, Italy. His current research interests include manipulator inverse kinematics techniques, redundant manipulator control, cooperative robot systems, and analysis and control of discrete-event systems. He has authored or coauthored 30 international journal papers, 73 international conference papers, four textbooks, and is a coeditor of the book Complex Robotic Systems (Springer-Verlag, 1998).

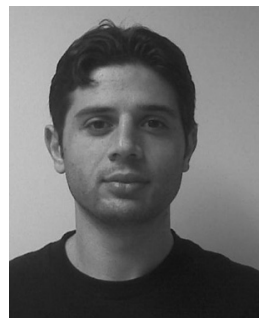

Alessandro Marino was born in Potenza, Italy, on March 1, 1982. He received the Laurea degree in computer engineering from the University of Naples Federico II, Naples, Italy, in 2006. He is currently working toward the $\mathrm{Ph} . \mathrm{D}$. degree in the Dipartimento di Ingegneria e Fisica dell'Ambiente, University of Basilicata, Potenza.

From April to October 2006, he worked under a research contract in the Dipartimento di Informatica e Sistemistica, University of Naples Federico II. From April to September 2008, he was a Visiting Scholar in the Department of Computer Science, University of Tennessee, Knoxville. His current research interests include cooperative robot manipulation, trajectory planning, and control algorithms for platoons of cooperative robots moving in an unknown environment. He is the author of two conference papers and one journal paper.

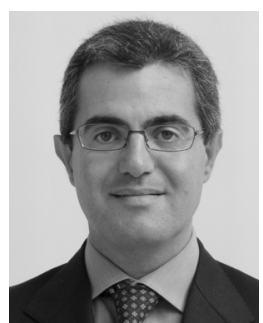

Luigi Villani (S'94-M'96-SM'03) was born in Avellino, Italy, on December 5, 1966. He received the Laurea degree in electronic engineering and the Research Doctorate degree in electronic engineering and computer science from the University of Naples, Naples, Italy, in 1992 and 1996, respectively.

$\mathrm{He}$ is currently an Associate Professor of automatic control in the Department of Computer and Systems Engineering, University of Naples. His current research interests include force/motion control of manipulators, cooperative robot manipulation, lightweight flexible arms, adaptive and nonlinear control of mechanical systems, visual servoing, fault diagnosis, and fault tolerance for dynamical systems. He has authored or coauthored three books, 35 journal papers, and 70 conference papers and book chapters.

Dr. Villani is an Associate Editor on the Conference Editorial Board of the IEEE Control Systems Society, and also an Associate Editor of the IEEE TRANSACTIONS ON CONTROL SYSTEMS TECHNOLOGY and IEEE TRANSACTIONS ON ROBOTICS. 